\title{
Sharing Clinical Notes in a Canadian Mental Health Setting: Recommendations from Applying the Consolidated Framework for Intervention Research
}

\author{
Brian LO a , Iman KASSAMa ${ }^{\text {a }}$ Keri DUROCHER ${ }^{\mathrm{a}}$, Danielle SHIN ${ }^{\mathrm{a}}$, \\ Nelson SHEN ${ }^{\mathrm{a}}$ and Gillian STRUDWICK ${ }^{\mathrm{a}}$ \\ ${ }^{a}$ Centre for Complex Interventions, Centre for Addiction and Mental Health
}

\begin{abstract}
During the COVID-19 pandemic, the OpenNotes movement presents an optimal solution for virtual engagement through the sharing of clinical notes within mental health care settings. Therefore, we conducted interviews to discover how mental health clinicians interact with patients using OpenNotes. We integrated The Consolidated Framework for Intervention Research to establish implementation recommendations. As both challenges and opportunities were identified, future research should address challenges to foster patient and clinician engagement in sharing clinical notes.
\end{abstract}

Keywords. Clinical notes, patient portal, mental health, OpenNotes, psychiatry, nursing informatics

\section{Introduction}

The COVID-19 pandemic has drastically changed how patients engage with clinicians, such as nurses, in the delivery of their mental health care $[1 ; 2]$. With social distancing restrictions in place, virtual opportunities for patients to engage with nurses is imperatively needed [3]. OpenNotes is one promising approach to do so and is the concept of sharing clinical documentation (e.g., admission notes, discharge summaries) through a patient portal. Current research has found favorable acceptability and interest from patients [4] and mental health care providers [5]. However, despite the interest in the concept, many of the expressed benefits remain largely hypothetical [6], and reluctance exists due to the fear of negative consequences associated with the sharing of clinical notes. As such, identifying recommendations and approaches to address the challenges and barriers observed with adopting OpenNotes is needed.

The Consolidated Framework for Intervention Research (CFIR) [7] is an implementation framework that supports the identification of recommendations to address observed barriers and encourage successful implementation. Numerous constructs related to the intervention, the setting (outer and inner), the individuals and the implementation process are outlined in this framework [7]. Careful consideration of challenges and barriers can provide an opportunity to explore how to improve implementation success for complex interventions such as OpenNotes. 
In this poster presentation, we applied the CFIR framework to identify recommendations for improving OpenNotes adoption among clinicians in a Canadian mental health setting. Given the critical role of nurses in supporting the delivery of mental health care, we believe that these findings will offer substantial value in accelerating the adoption of OpenNotes at the bedside.

\section{Methods}

This research aims to build on findings from a qualitative study that focused on the development of interventions to improve uptake of OpenNotes in mental health settings. In this study, one-to-one semi-structured interviews were conducted using a video conferencing platform among a variety of mental health clinicians (e.g., nurses, case managers, practice managers). We mapped our interview findings on to the CFIR framework, then brainstormed recommendations to help improve implementation success of OpenNotes in a Canadian mental health setting. We then mapped out the findings across the different constructs of the CFIR framework [7].

\section{Results}

\subsection{Intervention Characteristics}

Clinicians that deliver mental health care perceive a relative advantage of adapting OpenNotes within their organizations. An adaptability framework would be useful to suit the context of the care settings and patient populations, including long-term, outpatient, and acute care. Most stakeholders agree that trialability is imperative, with smaller scale implementation procedures proposed as an effective strategy to engage clinicians and patients in the OpenNotes movement.

\subsection{Outer Setting}

The needs of patients are imperative to consider when adopting the OpenNotes movement, with a focus on patients as collaborators in their care using recovery-oriented and narrative language. This key driver in OpenNotes adoption fosters a community organizational approach, defined within the CFIR as "cosmopolitanism."

\subsection{Inner Setting}

Considerations for structural characteristics and a positive culture shift are inherently important for OpenNotes adoption. Managerial support through leadership engagement and options for implementation pilots can help clinicians and patients feel confident in adapting note sharing practices. Relative priority of the OpenNotes movement should be emphasized within training programs to highlight the importance of sharing clinical notes with patients and the potential benefits. 


\subsection{Characteristics of Individuals}

Knowledge and beliefs about the OpenNotes movement should be gathered by project leaders to help clarify misconceptions and alleviate implementation concerns. Promoting self-efficacy through individualized training programs (webinars, podcasts, videos) will help clinicians achieve confidence in sharing notes with their patients. Review of clinician charting practices and providing guidance on therapeutic language choice will help clinicians successfully progress through the individual stages of change.

\subsection{Implementation Process}

Planning and engagement methods of implementation should focus on exemplars from organizations that have adopted OpenNotes into their care delivery. Training practice champions to support the sharing of clinical notes can help guide clinicians in this movement. Other successful execution methods can include integration into mandatory training requirements, organizational sessions or focus groups, and clinician education programs. Reflection and evaluation practices can also involve mitigation and auditing strategies to address conflicts.

\section{Conclusions}

Through application of the CFIR framework [7], several challenges and opportunities that may hinder the utility and adoption of OpenNotes in both community and hospital mental health organizations are identified. Future research should focus on implementing, evaluating, and optimizing possible interventions that can address the identified challenges. This information can guide nurse leaders and policymakers to make evidence-based decisions for the successful implementation of OpenNotes.

\section{References}

[1] Blease CR, O’Neill SF, Torous J, DesRoches CM, Hagglund M. Patient access to mental health notes: Motivating evidence-informed ethical guidelines. J Nerv Ment Dis 209 (2021), 265-269.

[2] Kameg BN. Psychiatric-mental health nursing leadership during Coronavirus Disease 2019 (COVID-19). J Psychiatr Ment Health Nurs (2020).

[3] Shore JH, Schneck CD, Mishkind MC. Telepsychiatry and the Coronavirus Disease 2019 pandemiccurrent and future outcomes of the rapid virtualization of psychiatric care. JAMA Psychiatry (2020).

[4] Peck P, Torous J, Shanahan M, Fossa A, Greenberg W. Patient access to electronic psychiatric records: A pilot study. Health Policy and Technology 6 (2017), 309-315.

[5] Dobscha SK, Denneson LM, Jacobson LE, Williams HB, Cromer R, Woods S. VA mental health clinician experiences and attitudes toward OpenNotes, Gen Hosp Psychiatry 38 (2016), 89-93.

[6] Chimowitz H, O’Neill S, Leveille S, Welch K, Walker J. Sharing psychotherapy notes with patients: Therapists' attitudes and experiences. Soc Work 65 (2020), 159-168.

[7] Damschroder LJ, Aron DC, Keith RE, Kirsh SR, Alexander JA, Lowery JC. Fostering implementation of health services research findings into practice: A consolidated framework for advancing implementation science. Implement Sci 4 (2009), 50. 